\title{
ON DENSE SUBSETS OF THE MEASURE ALGEBRA
}

\author{
J. CICHOŃ, A. KAMBURELIS AND J. PAWLIKOWSKI
}

\begin{abstract}
We show that the minimal cardinality of a dense subset of the measure algebra is the same as the minimal cardinality of a base of the ideal of Lebesgue measure zero subsets of the real line.
\end{abstract}

0. Introduction. Let $(P, \leqslant)$ be a given partial ordering. A subset $D \subseteq P$ is called dense if for any $p \in P$ there exists $d \in D$ such that $d \leqslant p$. A subset $D$ is called upward dense if $D$ is dense in $(P, \geqslant)$. Let $\Delta(P, \leqslant)$ denote the minimal cardinality of a dense subset of $(P, \leqslant)$.

Recall that with any boolean algebra $\mathscr{C}$ we can associate a natural partial ordering $\leqslant \mathscr{8}$. Let 0 and 1 denote the minimal and the maximal elements in this ordering. A dense or upward dense subset of $\mathscr{C}$ is a subset of $\mathscr{C} \backslash\{0,1\}$ which is dense or

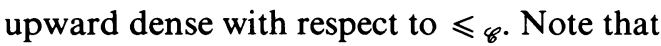

$$
\Delta\left(\mathscr{C} \backslash\{0,1\}, \leqslant_{\mathscr{C}}\right)=\Delta(\mathscr{C} \backslash\{0,1\}, \mathscr{\mathscr { C }} \geqslant) .
$$

We call this cardinal number $\Delta(\mathscr{C})$.

Let $\mathscr{I}$ be an ideal of subsets of a set $X$. A base of $\mathscr{I}$ is an upward dense subset of $(\mathscr{I}, \subseteq)$. Let $\Delta(\mathscr{I})=\Delta(\mathscr{I}, \supseteq)$. $\mathscr{I}$ is a $\sigma$-ideal if for any countable $\mathscr{A} \subseteq \mathscr{I}$ we have $\cup \mathscr{I} \in \mathscr{I}$. Let $\mathscr{I}^{c}$ denote the dual filter $\{X \backslash A: A \in \mathscr{I}\}$.

In this paper $\mathscr{B}$ denotes the $\sigma$-field of Borel subsets of the Cantor set $\omega_{2}$ ( $\omega$ denotes the set of natural numbers and 2 denotes the set $\{0,1\}$ ). Let $\mathscr{K}$ denote the ideal of subsets of ${ }^{\omega} 2$ of first Baire category. The canonical product measure on ${ }^{\omega} 2$ is called the Lebesgue measure on ${ }^{\omega} 2$. Let $\mathscr{L}$ denote the ideal of subsets of ${ }^{\omega} 2$ of Lebesgue measure zero. Note that both ideals $\mathscr{K}$ and $\mathscr{L}$ have Borel bases (i.e. bases contained in $\mathscr{B}$ ). If $\mathscr{I}$ is an ideal on ${ }^{\omega} 2$ then $\mathscr{I} \cap \mathscr{B}$ is an ideal in the $\sigma$-field $\mathscr{B}$. We denote the quotient boolean algebra $\mathscr{B} /(\mathscr{I} \cap \mathscr{B})$ by $\mathscr{B} / \mathscr{I}$.

A boolean algebra is $\sigma$-saturated if there is no uncountable family of pairwise disjoint elements of this algebra. Recall that the algebras $\mathscr{B} / \mathscr{K}$ and $\mathscr{B} / \mathscr{L}$ are $\sigma$-saturated. Suppose that $\mathscr{C}$ is a boolean algebra and $a \in \mathscr{C}$. Then by $\mathscr{C}_{a}$ we denote the boolean algebra with the universe $\left\{b \in \mathscr{C}: b \leqslant_{\mathscr{C}} a\right\}$ endowed with the operations canonically defined from the operations in $\mathscr{C}$.

1. The main result. We show a method of constructing a dense subset of the boolean algebra $\mathscr{B} / \mathscr{I}$ from a base of $\mathscr{I}$ for some class of ideals on ${ }^{\omega} 2$.

Received by the editors July $29,1983$.

1980 Mathematics Subject Classification. Primary 04A15; Secondary 28A05. 
THEOREM 1.1. Suppose $\mathscr{I}$ is a $\sigma$-ideal on ${ }^{\omega} 2$ with a Borel base and $\mathscr{B} / \mathscr{I}$ is $\sigma$-saturated. Then $\Delta(\mathscr{B} / \mathscr{I}) \leqslant \Delta(\mathscr{I}) \cdot \boldsymbol{\aleph}_{0}$.

Proof. For $n \in \omega$ let $I_{n}=\left\{f \in{ }^{\omega} 2: f(n)=0\right\}$. The family $\left\{I_{n}: n \in \omega\right\}$ o-generates $\mathscr{B}$ and has the following independence property: for any $\sigma$-complete Boolean algebra $\mathscr{C}$ and a function $f:\left\{I_{n}: n \in \omega\right\} \rightarrow \mathscr{C}$, there exists a $\sigma$-complete homomorphism $h: \mathscr{B} \rightarrow \mathscr{C}$ which extends $f$ (see [3, Theorem 31.6]).

For any $A \in \mathscr{B}$, we fix an ordinal $\alpha(A)<\omega$, and a sequence $\left\{B_{\xi}^{A}: \xi \leqslant \alpha(A)\right\}$ such that $B_{\alpha(A)}^{A}=A$, and for every $\xi \leqslant \alpha(A)$ we have $B_{\xi}^{A}={ }^{\omega} 2 \backslash B_{\eta}^{A}$ for some $\eta<\xi$, or $B_{\xi}^{A}=\bigcup\left\{B_{\eta}^{A}: \eta \in X\right\}$ for some $X \subseteq \xi$, or $B_{\xi}^{A}=I_{n}$ for some $n \in \omega$.

LeMMA 1.2. Suppose $\mathscr{J}$ is a $\sigma$-ideal on ${ }^{\omega} 2$ with a Borel base and $\mathscr{B} / \mathscr{J}$ is $\sigma$-saturated. Then there exists $a \in \mathscr{B} / \mathscr{J} \backslash\{0\}$ such that $\Delta\left((\mathscr{B} / \mathscr{J})_{a}\right) \leqslant \Delta(\mathscr{J}) \cdot \boldsymbol{\aleph}_{0}$.

Proof. Let $\mathscr{A} \subseteq \mathscr{B} \cap \mathscr{J}$ be a base of $\mathscr{J}$ of minimal cardinality. Let $\mathscr{C}_{0}$ be the subalgebra of $\mathscr{B}$ generated by $\left\{B_{\xi}^{A}: A \in \mathscr{A} \wedge \xi \leqslant \alpha(A)\right\} \cup\left\{\mathscr{I}_{n}: n \in \omega\right\}$. Then $\left|\mathscr{C}_{0}\right|$ $\leqslant \Delta(\mathscr{J}) \cdot \boldsymbol{\aleph}_{0}$.

Let $\mathscr{C}$ be the complete boolean algebra for which $\mathscr{C}_{0} / \mathscr{J}$ is a dense subalgebra and let $\pi: \mathscr{C}_{0} \rightarrow \mathscr{C}$ be the canonical embedding, i.e. $\pi(C)=[C]_{\mathscr{J}}$. Let $\tilde{\pi}: \mathscr{B} \rightarrow \mathscr{C}$ be the $\sigma$-homomorphism extending $\pi \uparrow\left\{I_{n}: n \in \omega\right\}$. For $A \in \mathscr{A}$ and $\xi \leqslant \alpha(A)$, one can prove by induction on $\xi$ that $\tilde{\pi}\left(B_{\xi}^{A}\right)=\pi\left(B_{\xi}^{A}\right)$. It follows that, for every $C \in \mathscr{C}_{0}$, we have $\tilde{\pi}(C)=\pi(C)$, i.e., $\tilde{\pi}$ extends $\pi$.

Let $\varphi: \mathscr{B} / \mathscr{J} \rightarrow \mathscr{C}$ be the $\sigma$-homomorphism defined by $\varphi\left([B]_{\mathscr{J}}\right)=\tilde{\pi}(B)$ for $B \in \mathscr{B}$. The definition is correct because $\mathscr{J} \subseteq \operatorname{ker} \tilde{\pi}$. Let $\mathscr{E} \subseteq \mathscr{B} \backslash \mathscr{J}$ be a maximal $\mathscr{J}$-disjoint family such that $\left\{[E]_{\mathscr{J}}: E \in \mathscr{E}\right\} \subseteq \operatorname{ker} \varphi(\mathscr{J}$-disjoint means that $E \cap F \in \mathscr{J}$ for any different elements of $\mathscr{E})$. Then $\mathscr{E}$ is countable, so $[\cup \mathscr{E}]_{\mathscr{J}} \in \operatorname{ker} \varphi$. Let $a=\left[{ }^{\omega} 2 \backslash \bigcup \mathscr{E}\right]_{\mathscr{J}}$. Then $a \neq 0$ and the algebras $(\mathscr{B} / \mathscr{J}) / \operatorname{ker} \varphi$ and $(\mathscr{B} / \mathscr{J})_{a}$ are isomorphic. Since $\varphi(\mathscr{B} / \mathscr{J})=\mathscr{C}$, the algebras $(\mathscr{B} / \mathscr{J})_{a}$ and $\mathscr{C}$ are isomorphic. $\mathscr{C}$ has a dense subset, the cardinality of which is $\Delta(\mathscr{J}) \cdot \boldsymbol{\aleph}_{0}$, so $(\mathscr{B} / \mathscr{J})_{a}$ does also. Hence, the lemma is proved.

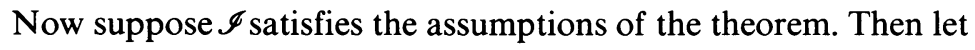

$$
\mathscr{A}=\left\{a \in \mathscr{B} / \mathscr{I} \backslash\{0\}: \Delta\left((\mathscr{B} / \mathscr{I})_{a}\right) \leqslant \Delta(\mathscr{I}) \cdot \boldsymbol{\aleph}_{0}\right\} .
$$

We show that $\mathscr{A}$ is dense in $\mathscr{B} / \mathscr{I}$. Suppose $[B]_{\mathscr{I}} \in \mathscr{B} / \mathscr{I} \backslash\{0\}$ and let $\mathscr{J}$ be the ideal generated by $\mathscr{I}$ and ${ }^{\omega} 2 \backslash B$. Then $\mathscr{B} / \mathscr{J}$ is $\sigma$-saturated and $\Delta(\mathscr{J}) \leqslant \Delta(\mathscr{I})$. By the lemma there exists $a^{\prime} \in \mathscr{B} / \mathscr{J} \backslash\{0\}$ such that $\Delta\left((\mathscr{B} / \mathscr{J})_{a}\right) \leqslant \Delta(\mathscr{J}) \cdot \boldsymbol{\aleph}_{0} \leqslant \Delta(\mathscr{I})$. $\boldsymbol{\aleph}_{0}$. Then there exists a Borel set $A \subseteq B$ such that $a^{\prime}=[A]_{\mathscr{J}}$. Let $a=[A]_{\mathscr{J}}$. Then the algebras $(\mathscr{B} / \mathscr{J})_{a^{\prime}}$ and $(\mathscr{B} / \mathscr{I})_{a}$ are isomorphic and $a \leqslant[B]_{\mathscr{I}}$. Hence, $\mathscr{A}$ is dense in $\mathscr{B} / \mathscr{I}$.

Now let $\mathscr{A}^{*}$ be a maximal subfamily of $\mathscr{A}$ consisting of pairwise disjoint elements. Then $\left|\mathscr{A}^{*}\right| \leqslant \boldsymbol{\aleph}_{0}$. For each $a \in \mathscr{A}^{*}$ let $\mathscr{D}_{a}$ be a dense subset of $(\mathscr{B} / \mathscr{I})_{a}$, the cardinality of which is at most $\Delta(\mathscr{I}) \cdot \boldsymbol{\aleph}_{0}$. Then $\bigcup\left\{\mathscr{D}_{a}: a \in \mathscr{A}^{*}\right\}$ is a dense subset of $\mathscr{B} / \mathscr{I}$ with cardinality at most $\Delta(\mathscr{I}) \cdot \boldsymbol{\aleph}_{0}$. Hence the theorem is proved.

2. Steinhaus property of ideals. It follows from Theorem 1.1 that $\Delta(\mathscr{B} / \mathscr{L}) \leqslant \Delta(\mathscr{L})$. We show that the reverse inequality is also true. 
Let $G$ be a group, $a \in G$ and $A, B \subseteq G$. We define $A^{-1}=\left\{x^{-1}: x \in A\right\}, A \cdot B=$ $\{x \cdot y: x \in A \wedge y \in B\}$ and $A \cdot a=\{x \cdot a: x \in A\}$.

Definition 2.1. Let $G$ be a group and $\mathscr{C}$ a $\sigma$-complete field of subsets of $G$. A $\sigma$-complete ideal $\mathscr{I}$ on $G$ satisfies the $\mathscr{C}$-Steinhaus property if there exist a base of $\mathscr{I}$ contained in $\mathscr{C}$ and a countable set $W \subseteq G$ such that

(i) $(\forall A \in \mathscr{I})(\forall q \in W)(A \cdot q \in \mathscr{I})$,

(ii) $(\forall A, B \in \mathscr{C} \backslash \mathscr{I})\left(\left(B^{-1} \cdot A\right) \cap W \neq \varnothing\right)$.

Two well-known ideals on ${ }^{\omega} 2$ which satisfy the $\mathscr{B}$-Steinhaus proeprty are $\mathscr{L}$ and $\mathscr{K}$ (see [2, Theorem 4.8]). We give some other examples. Suppose $\mathscr{I}$ and $\mathscr{J}$ are ideals on ${ }^{\omega} 2$. Then

$$
\mathscr{I} \times \mathscr{J}=\left\{X \subseteq{ }^{\omega} 2 \times^{\omega} 2:\{x:\{y:\langle x, y\rangle \in X\} \notin \mathscr{J}\} \in \mathscr{I}\right\} .
$$

Let $\mathscr{B}_{2}$ denote the $\sigma$-field of Borel subsets on ${ }^{\omega} 2 \times^{\omega} 2$. Then we define

$$
\mathscr{I} \otimes \mathscr{J}=\left\{X \subseteq{ }^{\omega} 2 \times{ }^{\omega} 2:\left(\exists Y \in \mathscr{B}_{2} \cap(\mathscr{I} \times \mathscr{J})\right)(X \subseteq Y)\right\} .
$$

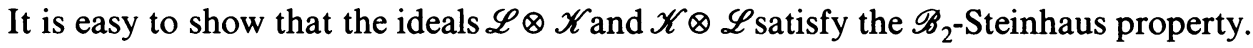

The next lemma clarifies condition (ii) of Definition 2.1.

LEMMA 2.2. Let $G$ be a group and $\mathscr{C}$ a $\sigma$-field of subsets of $G$. Let $\mathscr{I}$ be an ideal with a base contained in $\mathscr{C}$. Suppose $W$ is a countable subset of $G$ such that $(\forall A \in \mathscr{I})(\forall q \in$ $W)(A \cdot q \in \mathscr{I})$. Then the following sentences are equivalent:

(i) $(\forall A, B \in \mathscr{C} \backslash \mathscr{I})\left(\left(B^{-1} \cdot A\right) \cap W \neq \varnothing\right)$,

(ii) $(\forall A \in \mathscr{C} \backslash \mathscr{I})\left(A \cdot W^{-1} \in \mathscr{I}^{c}\right)$,

(iii) $\left(\forall A \in \mathscr{C} \backslash \mathscr{I}^{c}\right)\left(\cap\left\{A \cdot q^{-1}: q \in W\right\} \in \mathscr{I}\right)$.

Proof. (ii) $\Leftrightarrow$ (iii) is obvious. (i) $\Rightarrow$ (ii). Suppose $A \in \mathscr{C} \backslash \mathscr{I}$ and $A \cdot W^{-1} \notin \mathscr{I}^{c}$. Then there exists $B \in \mathscr{C} \backslash \mathscr{I}$ such that $\left(A \cdot W^{-1}\right) \cap B=\varnothing$. But then $\left(B^{-1} \cdot A\right) \cap$ $W=\varnothing$.

(ii) $\Rightarrow$ (i). Suppose $A, B \in \mathscr{C} \backslash \mathscr{I}$. Then by (ii) we have $\left(A \cdot W^{-1}\right) \in \mathscr{I}^{c}$; hence, $\left(A \cdot W^{-1}\right) \cap B \neq \varnothing$, so $\left(B^{-1} \cdot A\right) \cap W \neq \varnothing$.

The following lemma is an application of the Steinhaus property.

Lemma 2.3. Suppose $\mathscr{C}$ is a $\sigma$-field on a group $G$. If the ideal $\mathscr{I}$ on $G$ satisfies the $\mathscr{C}$-Steinhaus property, then $\Delta(\mathscr{I}) \leqslant \Delta(\mathscr{C} \backslash \mathscr{I})$.

Proof. Let $\mathscr{A}$ be a dense subset of $\left(\mathscr{C} \backslash \mathscr{I}^{c}, \supseteq\right)$, the cardinality of which is $\Delta\left(\mathscr{C} \backslash \mathscr{I}^{c}, \supseteq\right)$. Let $W$ be a countable subset of $G$ given by the Steinhaus property. Let $\mathscr{A}^{*}=\left\{\cap\left\{A \cdot q^{-1}: q \in W\right\}: A \in \mathscr{A}\right\}$. Then $\left|\mathscr{A}^{*}\right| \leqslant|\mathscr{A}|$ and $\mathscr{A}^{*} \subseteq \mathscr{I}$. We show that $\mathscr{A}^{*}$ is a base of $\mathscr{I}$. Let $B \in \mathscr{I}$. Then $B \cdot W \in \mathscr{I}$. Hence, there exists $A \in \mathscr{A}$ such that $B \cdot W \subseteq A$. Then $B \subseteq \bigcap\left\{A \cdot q^{-1}: q \in W\right\}$. Hence, the lemma is proved.

The next lemma gives us a lower estimation of $\Delta(\mathscr{B} / \mathscr{L})$.

LEMMA 2.4. $\Delta(\mathscr{B} \backslash \mathscr{L}, \subseteq) \leqslant \Delta(\mathscr{B} / \mathscr{L})$.

Proof. Let $\mathscr{A}$ be a dense subset of $\mathscr{B} / \mathscr{L} \backslash\{0\}$ with cardinality $\Delta(\mathscr{B} / \mathscr{L})$. We may assume $\mathscr{A}=\left\{[A]: A \in \mathscr{A}^{*}\right\}$ and, for each $A \in \mathscr{A}^{*}$ and every open set $U$, if $A \cap U \neq \varnothing$, then $A \cap U \notin \mathscr{L}$. We show that $\mathscr{A}^{*}$ is dense in $(\mathscr{B} \backslash \mathscr{L}, \subseteq)$. Suppose $B \in \mathscr{B} \backslash \mathscr{L}$. Let $C \subseteq B$ be a closed set such that $C \notin \mathscr{L}$. Then there exists $A \in \mathscr{A}^{*}$ such that $[A] \leqslant[C]$. But ${ }^{\omega} 2 \backslash C$ is an open set, hence $A \subseteq C$. 
Note that $\Delta(\mathscr{B} \backslash \mathscr{I}, \subseteq)=\Delta\left(\mathscr{B} \backslash \mathscr{I}^{c}, \supseteq\right)$. Hence from Theorem 1.1 and Lemmas 2.3 and 2.4 we obtain

THEOREM 2.5. $\Delta(\mathscr{B} / \mathscr{L})=\Delta(\mathscr{L})$.

3. Generalizations and comments. (1) We show that in the formulation of Theorem 1.1 the assumption of the existence of a Borel base is essential.

THEOREM 3.1. If the theory ZFC is consistent, then the theory $Z F C+2^{\boldsymbol{N}_{0}}=\boldsymbol{\aleph}_{2}$ + "there exists a $\sigma$-ideal $\mathscr{I}$ on ${ }^{\omega} 2$ such that $\mathscr{B} / \mathscr{I}$ is $\sigma$-saturated, $\Delta(\mathscr{B} / \mathscr{I})=\boldsymbol{\aleph}_{2}$ and $\Delta(\mathscr{I})=\aleph_{1}$ " is consistent.

Proof. Let us add to a model of ZFC $+2^{\aleph_{0}}=\boldsymbol{\aleph}_{1}$, by means of a generic extension, a sequence $\left\{r_{\alpha}: \alpha<\omega_{2}\right\}$ of independent random reals. Let $X=\left\{r_{\alpha}: \alpha<\right.$ $\left.\omega_{1}\right\}$. Then for every set $A \in \mathscr{L}$, we have $|A \cap X| \leqslant \boldsymbol{\aleph}_{0}$.

Let $\mathscr{J}=\left\{A \subseteq{ }^{\omega} 2:|A \cap X| \leqslant \boldsymbol{\aleph}_{0}\right\}$. Then $\Delta(\mathscr{J})=\boldsymbol{\aleph}_{1}$. Let $\mathscr{A}$ be a maximal family of pairwise disjoint sets from $\mathscr{B} \backslash \mathscr{L}$ such that for every $A \in \mathscr{A}$ we have $|X \cap A| \leqslant \boldsymbol{\aleph}_{0}$. We put $B={ }^{\omega} 2 \backslash \cup \mathscr{A}$ and $b=[B]_{\mathscr{L}}$. It is easy to see that for every Borel set $C \subseteq B$ we have $(C \in \mathscr{L}$ iff $C \in \mathscr{J})$. From this we conclude that $\mathscr{B} / \mathscr{J} \cong(\mathscr{B} / \mathscr{L})_{b}$; hence

$$
\Delta(\mathscr{B} / \mathscr{J})=\Delta\left((\mathscr{B} / \mathscr{L})_{b}\right)=\Delta(\mathscr{B} / \mathscr{L})=\Delta(\mathscr{L}) .
$$

In our model, ${ }^{\omega} 2$ is not a union of less than $\aleph_{2}$ sets from $\mathscr{L}$. This fact implies that $\Delta(\mathscr{L})=\boldsymbol{\aleph}_{2}$.

(2) Let us consider ${ }^{\kappa} 2$ for a given infinite cardinal number $\kappa$. Let $\mathscr{B}_{\kappa}$ be the $\sigma$-algebra generated by $\left\{I_{\xi}: \xi<\kappa\right\}$, where $I_{\xi}=\left\{f \in{ }^{\kappa} 2: f(\xi)=0\right\}$. A slight modification of the proof of Theorem 1.1 gives us the following result.

THEOREM 3.2. Suppose $\mathscr{I}$ is a $\sigma$-ideal on ${ }^{\omega} 2$ with a base contained in $\mathscr{B}_{\kappa}$ such that $\mathscr{B}_{\kappa} / \mathscr{I}$ is $\sigma$-saturated. Then $\Delta\left(\mathscr{B}_{\kappa} / \mathscr{I}\right) \leqslant \Delta(\mathscr{I}) \cdot \boldsymbol{\aleph}_{0}$.

Let us consider the product measure on ${ }^{\kappa} 2$ arising from the measure on $\{0,1\}$ which gives values 0.5 to 0 and 1 . Let $\mathscr{L}_{\kappa}$ be the ideal of zero measure subsets of ${ }^{\kappa} 2$. Let $e_{\xi} \in{ }^{\kappa} 2$ be a function defined by $e_{\xi}(\xi)=1$ and $e_{\xi}(\eta)=0$ for $\eta \neq \xi$. For $B \in \mathscr{B}_{\kappa}$ we define $\operatorname{supp}(B)=\left\{\xi \in \kappa: B+e_{\xi} \neq B\right\}$ (we treat ${ }^{\kappa} 2$ here as a product of $\kappa$ copies of the cyclic group $\{0,1\}$ ).

Note that for any $B \in \mathscr{B}_{\kappa}$ we have $|\operatorname{supp}(B)| \leqslant \boldsymbol{\aleph}_{0}$. For any finite function $\sigma$, the domain of which is contained in $\kappa$ and range in $\{0,1\}$, let $\langle\sigma\rangle=\left\{f \in{ }^{\kappa} 2: \sigma \subseteq f\right\}$. Now suppose $A \in \mathscr{B}_{\kappa}$. We define $A^{*}=A \backslash \bigcup\left\{\langle\sigma\rangle:\langle\sigma\rangle \cap A \in \mathscr{L}_{\kappa} \wedge\right.$ domain of $\sigma$ is contained in $\operatorname{supp}(A)\}$.

Note that for every $A \in \mathscr{B}_{\kappa}$ we have $[A]_{\mathscr{L}_{\kappa}}=\left[A^{*}\right]_{\mathscr{L}_{\kappa}}$, and for every open set $U$, if $A \cap U \neq \varnothing$, then $A \cap U \notin \mathscr{L}_{\kappa}$.

We can now imitate the proof of Lemma 2.2 to obtain the following inequality.

LEMMA 3.3. $\Delta\left(\mathscr{B}_{\kappa} \backslash \mathscr{L}_{\kappa}, \subseteq\right) \leqslant \Delta\left(\mathscr{B}_{\kappa} / \mathscr{L}_{\kappa}\right)$.

Suppose $A \in \mathscr{B}_{\kappa} \backslash \mathscr{L}_{\kappa}^{c}$. Then the $\operatorname{set} \bigcap\left\{A+f: f \in{ }^{\kappa} 2 \wedge f^{-1}(\{1\})\right.$ is a finite subset of $\operatorname{supp}(A)\}$ belongs to $\mathscr{L}_{\kappa}$. This observation allows us to generalize the proof of Lemma 2.3: 
LEMMA 3.4. $\Delta\left(\mathscr{L}_{\kappa}\right) \leqslant \Delta\left(\mathscr{B}_{\kappa} \backslash \mathscr{L}_{\kappa}\right)$.

From the above lemmas and theorem we obtain the following equality.

COROLlaRy 3.5. For any infinite cardinal number $\kappa, \Delta\left(\mathscr{L}_{\kappa}\right)=\Delta\left(\mathscr{B}_{\kappa} / \mathscr{L}_{\kappa}\right)$.

(3) A precise estimation of $\Delta(\mathscr{L})$ is impossible. Clearly $\aleph_{1} \leqslant \Delta(\mathscr{L}) \leqslant 2^{\aleph_{0}}$. Since the additivity of Lebesgue measure is less than or equal to $\Delta(\mathscr{L})$, Martin's Axiom implies that $\Delta(\mathscr{L})=2^{\aleph_{0}}$. The theory ZFC $+2^{\aleph_{0}}+\boldsymbol{\aleph}_{2}+\Delta(\mathscr{L})=\boldsymbol{\aleph}_{1}$ is also (relatively) consistent (see [1]).

\section{REFERENCES}

1. J. Cichon, On bases of ideals and boolean algebras, Proc. Open Days for Model Theory and Set Theory (Jadwisin, 1981), edited by W. Guzicki, W. Marek, A. Pelc and C. Rauszer.

2. J. G. Oxtoby, Measure and category, Springer, Berlin, 1970.

3. R. Sikorski, Boolean algebras, Springer, Berlin, 1964.

Instytut Matematyki, Uniwersytetu Wroclawskiego, Pl. Grunwaldzki 2 / 4, 50 - 384 Wro Claw, Poland 